\title{
Integrated science of movement
}

\author{
Urška Demšar ${ }^{1}$, Jed A. Long ${ }^{2}$, and Katarzyna Siła-Nowicka ${ }^{3,4,5}$ \\ ${ }^{1}$ School of Geography \& Sustainable Development, University of St Andrews, UK \\ ${ }^{2}$ Department of Geography \& Environment, Western University, Canada \\ ${ }^{3}$ School of Environment, The University of Auckland, New Zealand \\ ${ }^{4}$ Urban Big Data Centre, University of Glasgow, Glasgow, Scotland, UK \\ ${ }^{5}$ Institute of Geodesy and Geoinformatics, Wrocław University of Environmental and Life Sciences, \\ Wrocław, Poland
}

\begin{abstract}
Recent technological advances in movement data acquisition have enabled researchers in many disciplines to study movement at increasingly detailed spatial and temporal scales. Yet there is little overlap in the sharing of methods and models between disciplines, despite similar research objectives and data models. Attempts to bridge this gap are leading towards the establishment of an overarching interdisciplinary science, termed the Integrated Science of Movement. Here we present opportunities and challenges of this process and outline the crucial role that GIScience as a discipline with a focus on space, place, and time can play in the integrated science of movement.
\end{abstract}

Keywords: movement analytics, human mobility, movement ecology, transportation, movement behavior

\section{Introduction}

Movement is a basic property of human and animal behavior and is studied across many disciplines. The study of animal movement is termed movement ecology, and its focus is to understand competition for resources and interaction between individuals and species [23]. Ecologists pioneered animal tracking technology over 70 years ago and these techniques are firmly embedded in contemporary ecology. In contrast, studies of human mobility are found across many disciplines: Geographic Information Science (GIScience), quantitative geography, transportation science, computer science, physics, and public health have all contributed to study of human mobility.

Methodologically, there is a high degree of similarity in concepts, data, and methods between disciplines; yet there exists a striking lack of interdisciplinary awareness that 
many problems (and solutions!) overlap. This has led to calls to bridge this gap, both in terms of establishing a theoretical data science framework for movement [10] and in terms of converging theories and concepts from the animal and human domains into a new discipline-termed the integrated science of movement [21]. This will require efforts to move out of our respective disciplinary silos and build connections, a process that is already starting through interdisciplinary collaborations. We argue that GIScientists are in a unique position to support the development of the integrated science of movement through our linkages across disciplines.

\section{Opportunities and challenges}

\subsection{Big data opportunities: new sources and data fusion}

Movement data are increasingly combined with data generated by different sensors on a tracking device. Co-located sensors measure properties of movement (e.g., accelerometers, magnetometers, gyrometers) and of the state of the environment (e.g., air temperature, salinity, wind strength) [32]. Other sensors provide data on interaction between individuals (e.g., proximity loggers in COVID-tracking apps [6]). In human mobility, wearable devices (fitness watches), tracking implants, and physiological sensors can provide data on the physical and emotional state of the person [7]. While locational measurements are the basis of answering questions such as where, how, and when the object is moving, colocated sensors allow researchers to answer more complex questions, such as identifying factors which influence movement behavior [32]. These sensors increase the volume and complexity of data and pose new analytical challenges-new methods and data models are needed to holistically represent and analyse movement data alongside co-located sensor data.

Environmental data from remote sensing or from smart urban sensors are now also widely used in movement analysis. Data experiments that combine movement, sensor and environmental data are becoming an essential tool [4,26]. Linking environmental and movement data introduces the problem of scale, as the spatial and temporal resolution of movement data rarely aligns with the spatial and temporal resolution of ancillary datasets about the environment. New methodological approaches are needed to study ways to find the appropriate scale for combining movement and environmental data in a way that preserves the accuracy of environmental information at the location and time of the moving object. Current solutions include multi-source data fusion [3], but new solutions are needed for unusual and geometrically complex environmental data (e.g., satellite data on Earth's magnetic field, dynamic traffic congestion data).

\subsection{Big data problems: geoprivacy}

An integrated science of movement has potential for finding solutions to the most pressing global problems—such as human contact tracing during the COVID-19 pandemic [6]which, however, requires wide and open access to movement data [27]. Movement ecology is at the forefront of this open data culture, as ecologists regularly share data online (see Movebank.org, Motus.org). Human mobility research has not reached the same level of data openness, owing to sensitive nature of individual movement data. Unfortunately, at present, methods for anonymising or preserving individual privacy are still in their in-

www.josis.org 
fancy [14]. At present, no anonymisation method is able to both provide sufficient privacy protection while maintaining the utility of anonymised data [27]. There are three possible paths forward to address this conflict. First, we can draw on the concept of differentially private environment [17], where aggregated data in form of collective movement patterns do not provide information about any individual. Second, we can combine the first concept with generalisation of sensitive attributes [8]. Third, we can use individual and collective simulations to create representative synthetic movement data, while not releasing real data; thus, safeguarding privacy [28].

\subsection{Big models: machine learning and artificial intelligences}

Modern movement data sets are large, and many cannot be analysed using traditional methods. This has led to an increase in the use of machine learning (ML) and artificial intelligence (AI) methods. While ML/AI for movement is not new (see [2]), methods such as deep learning have recently become popular [12]. ML/AI methods are excellent for narrow tasks that require fast analysis of a large amount of data (e.g., to predict individual movement from videos of animals or pedestrians [30,33]). A downside of their applications is that in many ML/AI studies (in particular in human mobility) these tools are applied without a deep understanding of the application domain. A further issue is that ML/AI methods replicate biases present in data and subsequently disadvantage specific individuals/groups [29]. A final common theme in ML/AI is that these new, computationally demanding and therefore also environmentally costly models only provide a marginal improvement against more established methods.

We do not discourage the use of ML/AI for movement research, but we emphasise the importance of careful consideration of the following. Like other methodologically focused studies, it is crucial that ML/AI movement researchers establish collaborations with domain experts to extract domain-relevant inferences from the results. The trend of performing the newest method on an old data set (e.g., those openly available on the internet) and (over)claim significant results has yet to produce new or exciting insights about either human or animal movement. Future work should therefore strive to make interdisciplinary connections to leverage the opportunities ML/AI methods provide and real questions in movement analysis. Movement research should also ensure to follow the ethical principles of ML/AI [20].

\subsection{Big problems require interdisciplinary collaborations}

Studying how people or animals make movement decisions in response to environmental conditions is inherently complex and this requires interdisciplinary collaborations between animal and human movement researchers [21,32]. A particularly relevant example at this moment in time is the global collaboration on wildlife-human interaction during COVID19 lockdown in the period of the so-called anthropause [25]. This initiative brings together ecologists, data scientists, and human mobility experts from over 300 teams from across the world to explore how animals have reacted to wide-scale changes in people's movements during the COVID-19 pandemic. Countries with extreme lockdown (China, Italy, Spain) registered less air pollution, less noise and less human presence to which animals responded by invading urban spaces. In other countries (Germany, Scandinavian countries), people were encouraged to take daily exercise in parks, which resulted in more frequent 
contact between wildlife and people. This initiative will link animal tracking data, human data (e.g., data from Strava), and environmental data (e.g., satellite data on air pollution, data on traffic-induced seismic noise [19]) to explore global changes in interactions between animals and humans.

\section{Opportunities for GIScience}

\subsection{Places and pathways}

Integrated science of movement is the study of the places people and animals visit and the pathways they use to move between them. These simple concepts underlie many of the modern movement models that are used to separate movement data into sequences of stops (which are associated with places) and more active movement periods (associated with pathways) $[5,15]$. To date, we have made much more progress using movement data to understand pathways-which is not surprising given that the reason we often collect movement data is to understand the movement part of the activity. However, significant opportunities exist for taking a more place-based emphasis, to explore the places individuals visit [31] and the sequences in which they are visited [3].

\subsection{Timing space and spacing time}

Movement is an inherently spatio-temporal process, yet most modern methodologies focus strictly on either of space or time. In movement, there is nothing spatial that is not temporal, but many movement studies have traditionally ignored this (studies of animal home ranges and human activity spaces being typical examples where time is ignored); although this is slowly changing $[9,22])$. Other examples of explicitly considering space and time as one single entity include capturing spatio-temporal patterns of co-occurrence [16] and finding repeating patterns of movement in space and/or time [13]. With its long-term tradition of spatio-temporal analysis, GIScience can lead in the integrated science of movement, to develop more robust and scalable spatio-temporal measures that can be applied across both animal and human movement research.

\subsection{Scales of movement}

Patterns of movement manifest across different spatial and temporal scales [23]. Further, our ability to capture these patterns is dependent on the spatial and temporal scale at which we collect, represent, and analyse movement data [18]. A well-known problem in regional geographical analysis is the modifiable areal unit problem (MAUP [24]) which shows that analysis performed at different spatial scales or using different aggregation shapes can lead to fundamentally different insights. While an integrated science of movement places emphasis on individual movement, it does not mean that similar aggregation effects do not exist for human mobility. Similarly, in movement ecology, aggregation of movement data across different spatio-temporal scales of an individual's lifetime track can lead to different inferences about resource use and competition [23]. GIScience theories of scale can contribute to better understand the scales of human [1] and wildlife [11] movement, and how our analysis choices influence patterns and the inferences we make from them.

www.josis.org 


\section{Conclusions}

We live at an exciting moment in time where we are effortlessly creating increasingly complex data on movement and environmental conditions that affect movement. There is a need for these data to be integrated and analysed together, which creates big and complex data science challenges. We, as GIScientists, are, through our understanding of space, time, scale and spatio-temporal processes, uniquely placed to help solve these challenges. Through this we can provide the missing link between animal and human movement research and contribute to establishment of the integrated science of movement.

\section{Acknowledgments}

Urška Demšar is supported by the Leverhulme Trust Research Project Grant (RPG-2018258). Jed Long is supported by a discovery grant from the Natural Sciences and Engineering Research Council of Canada.

\section{References}

[1] Alessandretti, L., Aslak, U., And Lehmann, S. The scales of human mobility. Nature 587, 7834 (2020), 402-407. doi:10.1038/s41586-020-2909-1.

[2] BLACK, W. Spatial interaction modeling using artificial neural networks. Journal of Transport Geography 3, 3 (1995), 159-166.

[3] Brum Bastos, V., Long, J., Church, K., Robson, G., De PaOla, R., And DEMŠAR, U. Multi-source data fusion of optical satellite imagery to characterize habitat selection from wildlife tracking data. Ecological Informatics 60 (2020), 101149. doi:10.1016/j.ecoinf.2020.101149.

[4] Brum Bastos, V., LONG, J., AND DemŠAR, U. Weather effects on human mobility: an analysis using multi-channel sequence analysis. Computers, Environment and Urban Systems 71 (2018), 131-152. doi:10.1016/j.compenvurbsys.2018.05.004.

[5] Buchin, M., Driemel, A., Van Kreveld, M., And Sacristan, V. Segmenting trajectories: A framework and algorithms using spatiotemporal criteria. Journal of Spatial Information Science 3, 3 (2011), 33-63. doi:10.5311/JOSIS.2011.3.66.

[6] Cho, H., Ippolito, D., AND YU, Y. Contact tracing mobile apps for COVID-19: Privacy considerations and related trade-offs. ArXiv (2020), arXiv:2003.11511.

[7] Chung, M., Fortunato, G., And RAdacsi, N. Wearable flexible sweat sensors for healthcare monitoring: a review. Journal of the Royal Society Interface 16 (2019), 20190217. doi:10.1098/rsif.2019.0217.

[8] Deldar, F., AND AbAdi, M. PDP-SAG: Personalized privacy protection in moving objects databases by combining differential privacy and sensitive attribute generalization. IEEE Access 7 (2019), 85887-902. doi:10.1109/ACCESS.2019.2925236. 
[9] DemšAr, U., AND LONG, J. Potential path volume (PPV): a geometric estimator for space use in 3D. Movement Ecology 7 (2019), 14. doi:10.1186/s40462-019-0158-4.

[10] Dodge, S. A data science framework for movement. Geographical Analysis (2019). doi:10.1111/gean.12212.

[11] Edwards, A., Phillips, R., Watkins, N., Freeman, M., Murphy, E., Afanasyev, V., BuldyreV, S., Da LuZ, M., RAPOso, E., Stanley, H., AND VisWANATHan, G. Revisiting levy flight search patterns of wandering albatrosses, bumblebees and deer. Nature 449 (2007), 1044-1049. doi:10.1038/nature06199.

[12] FAn, Z., SONG, X., XIA, T., JiAnG, R., SHIBASAKI, R., AND SAKURAMACHINS, R. Online deep ensemble learning for predicting citywide human mobility. Proceedings of the ACM on Interactive, Mobile, Wearable and Ubiquitous Technologies 2, 3 (2018), 105. doi:10.1145/3264915.

[13] FeUtrhaKe, U. Recognition of repetitive movement patterns-the case of football analysis. ISPRS International Journal of Geo-Information 5, 11 (2016), 208. doi:10.3390/ijgi5110208.

[14] Fiore, M., Katsikouli, P., Zavou, E., Cunche, M., Fessant, F., Hello, D., Aivodji, U., Olivier, B., Quertier, T., And StanicA, R. Privacy in trajectory micro-data publishing: A survey. ArXiv (2020), arXiv:1903.12211 [cs.CR].

[15] Gurarie, E., Bracis, C., Delgado, M., Meckley, T. D., Kojola, I., And WaGNER, C. M. What is the animal doing? tools for exploring behavioural structure in animal movements. Journal of Animal Ecology 85, 1 (2016), 69-84. doi:10.1111/13652656.12379 .

[16] Hoover, B. A., Miller, J. A., AND Long, J. Mapping areas of asynchronoustemporal interaction in animal-telemetry data. Transactions in GIS 24, 3 (2020), 573-586. doi:10.1111/tgis.12622.

[17] Kessler, C., And McKenZIE, G. A geoprivacy manifesto. Transactions in GIS 22, 1 (2018), 3-19. doi:10.1111/tgis.12305.

[18] Laube, P., AND PuRves, R. S. How fast is a cow? cross-scale analysis of movement data. Transactions in GIS 15, 3 (2011), 401-418. doi:10.1111/j.1467-9671.2011.01256.x.

[19] Lecocq, T., Hicks, S., Van Noten, K., Van Wijk, K., Koelemeijer, P., De Plaen, R., Massin, F., Hillers, G., Anthony, R., Apoloner, M., And ARroyo-SOLÓRZANO, M. Global quieting of high-frequency seismic noise due to COVID-19 pandemic lockdown measures. Science 369, 6509 (2020), 1338-1343. doi:10.1126/science.abd2438.

[20] Lo PIANO, S. Ethical principles in machine learning and artificial intelligence: cases from the field and possible ways forward. Nature Humanities and Social Sciences Communications 7, 9 (2020). doi:10.1057/s41599-020-0501-9.

[21] Miller, H., Dodge, S., Miller, J., AND Bohrer, G. Towards an integrated science of movement: converging research on animal movement ecology and human mobility science. International Journal of Geographical Information Science 33, 5 (2019), 855-876. doi:10.1080/13658816.2018.1564317.

www.josis.org 
[22] Miller, H. J., JAegal, Y., AND Raubal, M. Measuring the geometric and semantic similarity of space-time prisms using temporal signatures. Annals of the American Association of Geographers 109, 3 (2020), 730-753. doi:10.1080/24694452.2018.1484686.

[23] Nathan, R., Getz, W., Revilla, E., Holyoak, M., Kadmon, R., Saltz, D., AND SMOUSE, P. A movement ecology paradigm for unifying organismal movement research. Proceedings of the National Academy of Sciences 107, 49 (2008), 19052-19059. doi:10.1073/pnas.0800375105.

[24] Openshaw, S. The Modifiable Areal Unit Problem. GeoBooks, 1984.

[25] Rutz, C., Loretto, M., Bates, A., Davidson, S., Duarte, C., Jetz, W., Johnson, M., Kato, A., Kays, R., Mueller, T., AND Primack, R. COVID-19 lockdown allows researchers to quantify the effects of human activity on wildlife. Nature Ecology and Evolution 4 (2020), 1156-1159. doi:10.1038/s41559-020-1237-z.

[26] SiŁa-Nowicka, K., VAndrol, J., Oshan, T., LONG, J., DemŠAr, U., AND FotherINGHAM, S. Analysis of human mobility patterns from GPS trajectories and contextual information. International Journal of Geographic Information Science 30, 5 (2016), 881-906. doi:10.1080/13658816.2015.1100731.

[27] SMOLAK, K., RoHm, W., AND SiŁA-NowicKA, K. Urban-scale population mobility modelling for mobile phone data simulation. Computers, Environment and Urban Systems 84 (2020), 101526. doi:10.1016/ j.compenvurbsys.2020.101526.

[28] SmolaK, K., SiŁA-Nowicka, K., AND Rohm, W. Towards anonymous mobility data through the modelling of spatial circadian rhythms. In Proceedings of the 15th International Conference on Location Based Services (LBS 2019) (2019), pp. 117-23. doi:10.34726/lbs2019.

[29] VAN NOORDEN, R. The ethical questions that haunt facial-recognition research. $\mathrm{Na}$ ture 587 (2020), 354-358. doi:10.1038/d41586-020-03187-3.

[30] WALTER, T., AND COUZIN, I. TRex, a fast multi-animal tracking system with markerless identification, and 2D estimation of posture and visual fields. BioRxiv (2020).

[31] Westerholt, R., MocniK, F.-B., And Comber, A. A place for place: Modelling and analysing platial representations. Transactions in GIS 24, 4 (2020), 811-818. doi:10.1111/tgis.12647.

[32] Williams, H., Taylor, L., Benhamou, S., Bijleveld, A., Clay, T., De GrissaC, S., DEMŠAR, U., ENGLish, H., FranCONI, N., GÓMEZ-LAICH, A., AND GRIFFITHS, R. Optimizing the use of biologgers for movement ecology research. Journal of Animal Ecology 89, 1 (2020), 186-206. doi:10.1111/1365-2656.13094.

[33] XU, Y., PIAO, Z., AND GAO., S. Encoding crowd interaction with deep neural network for pedestrian trajectory prediction. In Proceedings of the IEEE/CVF Conference on Computer Vision and Pattern Recognition (2018), pp. 5275-5284. doi:10.1109/CVPR.2018.00553. 